\title{
Trajectory-tracking and Path-following Controllers for Constrained Underactuated Vehicles using Model Predictive Control*
}

\author{
Andrea Alessandretti ${ }^{1}{ }^{2}$, A. Pedro Aguiar ${ }^{3}$ and Colin N. Jones ${ }^{1}$
}

\begin{abstract}
This paper addresses the design of Model Predictive Control (MPC) laws to solve the trajectory-tracking problem and the path-following problem for constrained underactuated vehicles. By allowing an arbitrarily small asymptotic tracking error, we derive MPC laws where the size of the terminal set is only limited by the size of the system constraints. In fact, for the case of unconstrained inputs, the terminal set can be neglected and the resulting MPC controllers provide a global solution to the addressed constrained motion control problems. Simulation results are presented where the proposed MPC controllers are applied to 2-D and to 3-D moving vehicles.
\end{abstract}

\section{INTRODUCTION}

The motion control of underactuated vehicles has been along the years an attractive topic because of the wide range of practical applications and the theoretical challenges associated with the control problem.

In spite of that and the numerous results published in the technical literature, where a large set of the proposed control algorithms are obtained via Lyapunov-based design techniques, only few methods explicitly consider the case of constrained inputs signals. This results in control laws that, in practice, are only applicable in a limited region where the control action, designed for the unconstrained vehicle, does not violate the system constraints. We reference [1]-[5] for semi-global and global solutions to the (unconstrained) trajectory-tracking problem.

Model predictive control, given its ability to explicitly handle constraints, represents a natural direction for the control of constrained systems. A common approach to solve the trajectory-tracking and the path-following problem consists in rewriting them as stabilization problem in a conveniently defined error space, and then use the classic tools for the design of stabilizing MPC laws, e.g., [6]-[10]. However, since the error dynamics are often time-varying, solving this control problem remains a difficult task and only few results have been presented in the literature. For an overview on the topic see, for instance, the work [11]. In [12], sufficient conditions for stability are presented and in [13]-[15] a local solution to the trajectory-tracking problem can be found.

*This work was supported by projects CONAV/FCT-PT [PTDC/EEACRO/113820/2009], MORPH [EU FP7 ICT 288704], and FCT [PEst-OE/EEI/LA0009/2011]. The first author benefited from grant SFRH/BD/51073/2010 of the Foundation for Science and Technology (FCT), Portugal.

${ }^{1}$ École Polytechnique Fédérale de Lausanne (EPFL), Lausanne, Switzerland.

${ }^{2}$ Institute for Systems and Robotics (ISR), Instituto Superior Tecnico (IST), Lisbon, Portugal.

${ }^{3}$ Faculty of Engineering, University of Porto (FEUP), Portugal.
Moreover, for the specific case of unicycle model see also [16]. As main drawback of these approaches, the proposed terminal set and the terminal cost are only locally defined, resulting in a potentially limited region of the attraction.

Motivated by these observations, in this paper we address the trajectory-tracking problem and the path-following problem for underactuated vehicles with constrained inputs, where the motion is considered in both 2-D and 3-D cases. Allowing an arbitrarily small asymptotic tracking error, we derive MPC controllers using the results from [17] together with a nonlinear auxiliary control law proposed in [1]. The resulting terminal set is only limited by the size of the system constraints, leading to global solutions for the case of unconstrained systems.

The remainder of this paper is organized as follows. In Section II the addressed motion control problems are defined. In Section III we recall some results from the literature, which are used in Section III for the design of the MPC control laws. Numerical results are shown in Section V, where a model of a wheeled robot (2-D case) and a model of an aero vehicle (3-D case) are considered. Section VI closes the paper with some conclusions.

\section{Problem Statement}

This section describes the model of an underactuated vehicle and formulate the motion control problems addressed.

Let $I$ be an inertial coordinate frame and $B$ be a body coordinate frame attached to the vehicle. The pair $(p(t), R(t)) \in S E(3)^{1}$ denote the configuration of the vehicle, position and orientation, where $R(t)$ is the rotation matrix from body to inertial coordinates. Now, let $(v(t), \Omega(\omega(t))) \in \operatorname{se}(3)^{1}$ be the twist that defines the velocity of the vehicle, linear and angular, where the matrix $\Omega(\omega(t))$ is the skew-symmetric matrix associated to the angular velocity $\omega(t):=\left(\omega_{1}(t), \omega_{2}(t), \omega_{3}(t)\right)^{\prime}$, defined as

$$
\Omega(\omega(t)):=\left(\begin{array}{ccc}
0 & -\omega_{3}(t) & \omega_{2}(t) \\
\omega_{3}(t) & 0 & -\omega_{1}(t) \\
-\omega_{2}(t) & \omega_{1}(t) & 0
\end{array}\right) \in \mathbb{R}^{3 \times 3} .
$$

The kinematic model of a vehicle moving in 3D space satisfies

$$
\dot{p}(t)=R(t) v(t), \quad \dot{R}(t) \quad=R(t) \Omega(\omega(t)) .
$$

\footnotetext{
${ }^{1}$ For a given $n \in \mathbb{N}, S E(n)$ denotes the Cartesian product of $\mathbb{R}^{n}$ with the group $S O(n)$ of $n \times n$ rotation matrices and $s e(n)$ denotes the Cartesian product of $\mathbb{R}^{n}$ with the space $s o(n)$ of $n \times n$ skew-symmetric matrices.
} 
In this paper we consider constrained underactuated vehicles where the control input

$$
u(t):=\left(v_{1}(t) \quad \omega^{\prime}(t)\right)^{\prime} \in \mathcal{U}
$$

with $v(t):=\left(\begin{array}{lll}v_{1}(t) & 0 & 0\end{array}\right)^{\prime} \in \mathbb{R}^{3}$, consists of only the forward and the angular velocity, and is constrained to lie inside the compact input constraint set $\mathcal{U} \subset \mathbb{R}^{4}$ that, e.g, represents the physical limits of the actuators. For the sake of simplicity, we drop the explicit dependence on time wherever clear from the context. For the 2-D case the same model (1) applies, where differently, $(p, R) \in S E(2),(v, \Omega(\omega)) \in s e(2)$ with $v:=\left(v_{1}, 0\right)^{\prime}$ and $\Omega(\omega):=\left(\begin{array}{cc}0 & -\omega \\ \omega & 0\end{array}\right) \in \mathbb{R}^{2 \times 2}$, and $u:=\left(\begin{array}{ll}v_{1} & \omega\end{array}\right)^{\prime} \in \mathcal{U} \subset \mathbb{R}^{2}$. We consider the following trajectory-tracking and path-following problems:

Problem 1 (Constrained Trajectory-tracking): Consider a constrained vehicle described by (1) and let $p_{d}(t)$, with $t \in[0, \infty)$, be a differentiable desired trajectory. Design a control law such that, as $t$ goes to infinity, the position of the vehicle converges and remains inside a tube, centered around $p_{d}(\cdot)$, that can be made arbitrarily thin, i.e., $\left\|p(t)-p_{d}(t)\right\|$ converges to a neighborhood of zero that can be made arbitrarily small.

Problem 2 (Constrained Path-following): Consider a constrained vehicle described by (1) and let $p_{d}(\gamma)$ be a differentiable desired path parametrized with the parameter $\gamma \in[0, \infty)$. Moreover, let $\dot{\gamma} \in \mathcal{G}$ be a virtual input constrained inside a compact set $\mathcal{G} \subset \mathbb{R}$. Design a control law for $u$ and $\dot{\gamma}$ such that, as $t$ goes to infinity, i) the position of the vehicle converges and remains inside a tube, centered around $p_{d}(\gamma)$, that can be made arbitrarily thin and ii) the parameter $\gamma$ asymptotically satisfies a desired speed assignment $\dot{\gamma}_{d} \in \mathcal{G}$, i.e., $\left\|\dot{\gamma}(t)-\dot{\gamma}_{d}(t)\right\|$ goes to zero.

\section{BACKGROUND}

This section contains the definition of the MPC optimization problem and our previous result [17] that illustrates how to design a stable MPC controller using a nonlinear auxiliary law that exponentially stabilizes the unconstrained system.

\section{A. MPC optimization problem}

Consider the dynamical system

$$
\dot{x}(t)=f(x(t), u(t)), \quad x(0)=x_{0}, \quad t \geq 0
$$

with

$$
x(t) \in \mathcal{X} \subseteq \mathbb{R}^{n}, \quad u(t) \in \mathcal{U} \subseteq \mathbb{R}^{m}, \quad t \geq 0
$$

where $x(t) \in \mathbb{R}^{n}$ and $u(t) \in \mathbb{R}^{m}$ are the state and the input at time $t$, respectively, and $\mathcal{X} \subseteq \mathbb{R}^{n}$ and $\mathcal{U} \subseteq \mathbb{R}^{m}$ are the state and input constraint sets, respectively. For a generic trajectory $x(\cdot)$ we denote by $x([0, T])$ the trajectory considered in the time interval $[0, T]$. The MPC optimization problem is defined as follows:

Definition 1 (MPC Problem): Let $T \in(0, \infty)$ be a given horizon length and $\bar{x}([0, T])$ and $\bar{u}([0, T])$ be a pair of state and input predicted trajectories that satisfy (2) with initial condition $\bar{x}(0)=z$. The open loop MPC problem denoted by $P_{T}(z)$ consists of finding the optimal control signal $\bar{u}^{*}([0, T])$ that solves the following optimization problem

$$
\begin{array}{rlrl}
J_{T}^{*}(z) & =\min _{\bar{u}([0, T])} J_{T}(z, \bar{u}([0, T])) & & \\
\text { s.t. } & & & \\
\dot{\bar{x}}(\tau) & =f(\bar{x}(\tau), \bar{u}(\tau)) & & \forall \tau \in[0, T] \\
\bar{x}(0) & =z, \bar{x}(T) \in \mathcal{X}_{f} & & \\
\bar{x}(\tau) & \in \mathcal{X}, \bar{u}(\tau) \in \mathcal{U} & \forall \tau \in[0, T]
\end{array}
$$

with $J_{T}(z, \bar{u}([0, T])):=\int_{0}^{T} l(\bar{x}(\tau), \bar{u}(\tau)) d \tau+F(\bar{x}(T))$. The notation $\bar{u}^{*}([0, T] ; z)$ is used wherever we want to make the dependence to the initial state $z$ explicit. The finite horizon cost $J_{T}(\cdot)$ is composed of the stage cost $l: \mathcal{X} \times \mathcal{U} \rightarrow \mathbb{R}_{\geq 0}$ and the terminal cost $F: \mathcal{X}_{f} \rightarrow \mathbb{R}_{\geq 0}$. Throughout this paper we denote by $k_{f}: \mathcal{X}_{f} \rightarrow \mathcal{U}$ an auxiliary control law defined over the terminal set $\mathcal{X}_{f}$. In a state feedback sampled-data MPC framework the optimization problem $P_{T}(z)$ is repeatedly solved at discrete sampling instants $t_{i}=i \delta, i \in \mathbb{N}_{0}$, with $z=x\left(t_{i}\right)$ and $0<\delta \leq T$, where $\delta$ is the sampling time of the MPC law defined as

$$
u(t)=k_{M P C}(x):=\bar{u}^{*}\left(t-t_{i} ; x\left(t_{i}\right)\right), \forall t \in\left[t_{i}, t_{i+1}\right) .
$$

\section{B. Stable MPC using a pre-existing Auxiliary Control Law}

We consider the following assumptions [17]:

Assumption 1: $f(\cdot)$ is locally Lipschitz continuous in the region of interest and satisfies $f(0,0)=0$.

Assumption 2: The set $\mathcal{U} \subset \mathbb{R}^{m}$ is compact, $\mathcal{X} \subseteq \mathbb{R}^{n}$ is connected, and $(0,0) \in \operatorname{int}(\mathcal{X}) \times \operatorname{int}(\mathcal{U})^{2}$.

Assumption 3: The stage cost $l(\cdot)$ satisfies $l(0,0)=0$ and is lower bounded by a $\mathcal{K}_{\infty}$-class function ${ }^{3} w(\cdot)$, i.e., $w(\|x\|) \leq l(x, u), \forall(x, u) \in \mathcal{X} \times \mathcal{U}$.

Assumption 4: There exist a known control law $k_{f}: \mathcal{X}_{D} \rightarrow \mathbb{R}^{m}$ continuous around the origin and a continuously differentiable Lyapunov function $V(\cdot)$, and the positive constants $k_{1}, k_{2}, k_{3}$, and $a$ such that

$$
k_{1}\|x\|^{a} \leq V(x) \leq k_{2}\|x\|^{a}, \quad \frac{\partial V}{\partial x} f\left(x, k_{f}(x)\right) \leq-k_{3}\|x\|^{a}
$$

$\forall x \in \mathcal{X}_{D}$, for some set $\mathcal{X}_{D} \subseteq \mathbb{R}^{n}$ with $0 \in \operatorname{int}\left(\mathcal{X}_{D}\right) \subseteq \mathbb{R}^{n}$.

Assumption 5: The control law $k_{f}(\cdot)$ from Assumption 4 and the stage cost $l(\cdot)$ satisfy

$$
l\left(x, k_{f}(x)\right) \leq \sum_{i=1}^{v} a_{i}\|x\|^{i}, \quad \forall x \in \mathcal{X}_{l}
$$

for some set $\mathcal{X}_{l} \in \mathbb{R}^{n}$ with $0 \in \operatorname{int}\left(\mathcal{X}_{l}\right)$ and constants $v \in \mathbb{N}$, with $v \geq 1$, and $a_{i} \in \mathbb{R}, i=1, \ldots, v$, where $k_{1}, k_{2}, k_{3}$, and $a$ are positive constants.

Note that Assumption 5 is trivially verified for the classic case of a quadratic stage cost $l(x, u)=\|x\|_{Q}+\|u\|_{R}$, with $Q \in \mathbb{R}^{n \times n}, Q=Q^{T} \succeq 0$ and $R \in \mathbb{R}^{m \times m}$,

\footnotetext{
${ }^{2}$ Given a set $\mathcal{A}$, we denote by $\operatorname{int}(\mathcal{A})$ the interior of such set.

${ }^{3} \mathrm{~A}$ continuous function $w:[0, a) \rightarrow[0, \infty)$ is said to belong to class $\mathcal{K}$ if it is strictly increasing and $w(0)=0$. It is said to belong to class $\mathcal{K}_{\infty}$ if $a=\infty$ and $w(r) \rightarrow \infty$ as $r \rightarrow \infty$.
} 
$R=R^{T} \succeq 0$, and a linear auxiliary control law $u=K x$, with $K \in \mathbb{R}^{m \times n}$, where, for a given matrix $A, \lambda_{\min }(A)$ and $\lambda_{\max }(A)$ denote the smallest and largest real part of the eigenvalues of $A$, respectively, and for a given vector $a$ of suitable dimension, we use the notation $\|a\|_{A}^{2}=a^{\prime} A a$. In fact $l(x, K x) \leq\|x\|^{2} \lambda_{\max }\left(Q+K^{\prime} R K\right)$, which satisfies (5) with $v=2, a_{1}=0, a_{2}=\lambda_{\max }\left(Q+K^{\prime} R K\right)$, and $\mathcal{X}_{l}=\mathbb{R}^{n}$.

Moreover, it generalizes to the case where $l(\cdot)$ is upper bounded by a polynomial (without the constant term) in the state and in the input, and $k_{f}(\cdot)$ is upper bounded by a polynomial (without a constant term) in the state.

Theorem 1: Consider the constrained system (2) and the optimization problem $P_{T}(\cdot)$ with

$$
\begin{aligned}
F(x) & =\sum_{i=1}^{v} a_{i}\left(\frac{k_{2}}{k_{1}}\right)^{i / a} \frac{a k_{2}}{i k_{3}}\|x\|^{i} \\
\mathcal{X}_{f} & =\{x: V(x) \leq \alpha\} \subseteq \overline{\mathcal{X}}_{f},
\end{aligned}
$$

where $\overline{\mathcal{X}}_{f}:=\left\{x: x \in \mathcal{X} \cap \mathcal{X}_{D} \cap \mathcal{X}_{l}, k_{f}(x) \in \mathcal{U}\right\}$ and $\alpha>0$. If Assumptions 1-5 hold and $P_{T}(x(t))$ is feasible for $t=0$, then the origin of the resulting closed loop system (2) with (12) is asymptotically stable, with region of attraction consisting of the set of states $x \in \mathcal{X}$ for which $P_{T}(x)$ admits a feasible solution.

1) Computation of the Terminal Set: From [17], we recall a procedure to systematically compute the terminal set for the MPC scheme introduced above.

Let $\mathbb{R}[x]$ and $\Sigma[x]$ denote the set of polynomial and sunof-squares functions on $x$, respectively, with real coefficients.

Assumption 6: Suppose that the function $V(\cdot)$ is a polynomial function on $x$ and the set $\overline{\mathcal{X}}_{f}$ is a basic semi-algebraic set, i.e., it can be rewritten in the form

$$
\left\{x \in \mathbb{R}^{n}: q_{i}(x) \geq 0, q_{i} \in \mathbb{R}[x], i=1, \ldots, n_{q}\right\},
$$

for some polynomials $q_{i} \in \mathbb{R}[x], i=1, \ldots, n_{q}$ with $n_{q} \in \mathbb{N}$.

It can be shown that, the largest level value $\alpha$ such that condition (7) is satisfied is the solution to the following sumof-squares optimization problem:

$$
\begin{array}{rlrl}
\alpha^{*}:=\max _{\alpha, s_{1}, \ldots, s_{n_{q}}} \alpha & \\
\text { s.t. } & q_{i}-(\alpha-V) s_{i} \in \Sigma[x], & & \\
& s_{i} \in \Sigma[x], & i=1, \ldots, n_{q} \\
& & i=1, \ldots, n_{q}
\end{array}
$$

with $\operatorname{deg}\left(V s_{i}\right)=\operatorname{deg}\left(q_{i}\right), i=1, \ldots, n_{q}$, where for a given polynomial function $f \in \mathbb{R}[x] \operatorname{deg}(f)$ denotes the degree of the polynomial $f$. Although the optimization problem (9) cannot be directly solved using convex optimization methods, due to the product $\alpha s_{i}$ in (9b) that makes the problem non convex, it can be solved via bisection over the scalar variable $\alpha$. In fact, for any fixed $\alpha$, (9) becomes a convex feasibility problem, which can be efficiently solved using available toolboxes for sum-of-squares programming (e.g. SeDuMi [18], SDPT3 [19], YALMIP [20]).

Remark 1: The representation (8) is quite general, although, if the set $\overline{\mathcal{X}}_{f}$ can not be rewritten as (8), one could choose the set (8) to be an inner approximation of $\overline{\mathcal{X}}_{f}$. In this case, the proposed algorithm would return the largest level set of $V(\cdot)$ contained in the inner approximation.

\section{Main Results}

In this section, similarly to [1], we compute a trajectorytracking controller for the unconstrained model (1a), which is then used, together with Theorem 1, to design the desired MPC trajectory-tracking and path-following control laws.

Since we wish to control a vehicle with constrained inputs, some assumptions on the constraint sets $\mathcal{U}$ and $\mathcal{G}$ and on the desired vehicle velocity $v_{d}(t)$ are expected in order to guarantee that a solution to the control problem exists.

Assumption 7 (Bounded Desired Velocity): The desired vehicle velocity is bounded as $\left\|v_{d}(t)\right\| \leq \beta, \forall t \geq 0$, with $\beta \in \mathbb{R}_{\geq 0}$.

\section{A. Auxiliary Control Law}

Consider the error $e:=R^{\prime}\left(p-p_{d}\right)-\epsilon$, where $\epsilon$ is a given nonzero arbitrarily small constant vector of appropriate dimension. Note that as the error goes to zero the vehicle position converges to an arbitrarily thin tube, the $\epsilon$-tube, centered around $p(\cdot)$, i.e., $\left\|p-p_{d}\right\| \rightarrow\|\epsilon\|$ as $e \rightarrow 0$.

It is possible to show that

$$
\dot{e}=-\Omega e+\Delta u-R^{\prime} \dot{p}_{d}
$$

with

$$
\Delta=\left(\begin{array}{cc}
1 & \epsilon_{2} \\
0 & -\epsilon_{1}
\end{array}\right) \quad \text { or } \quad \Delta=\left(\begin{array}{cccc}
1 & 0 & -\epsilon_{3} & \epsilon_{2} \\
0 & \epsilon_{3} & 0 & -\epsilon_{1} \\
0 & -\epsilon_{2} & \epsilon_{1} & 0
\end{array}\right)
$$

for the 2-D case or 3-D case, respectively, where $\epsilon_{i}$ denotes the ith component of the vector $\epsilon$.

Proposition 1 (Trajectory-tracking controller): Consider the system (1a) in closed-loop with

$$
u=k\left(R, e, \dot{p}_{d}\right)=\bar{\Delta}\left(R^{\prime} \dot{p}_{d}-K e\right),
$$

where $\bar{\Delta}:=\Delta^{\prime}\left(\Delta \Delta^{\prime}\right)^{-1}, K$ is a given positive-definite matrix with suitable dimensions, and $\epsilon$ is such that $\Delta$ is full rank. Then, the origin $e=0$ of the closed-loop (10) with (11) is a global exponentially stable equilibrium point.

Proof: Consider the function $V=(1 / 2) e^{\prime} e$. Combining its derivative $\dot{V}=e \dot{e}=e^{\prime}\left(-\Omega e+\Delta u-R^{\prime} \dot{p}_{d}\right)$ with the control input (11) we obtain $\dot{V}=-e^{\prime}(\Omega+K) e=-e^{\prime} K e$, where we used the fact that $\Omega$ is a skew-symmetric matrix for which $x^{\prime} \Omega x=0, \forall x$ holds, which using standard Lyapunov theory, concludes the proof.

Note that Assumption 7 implies $k(\cdot, e, \cdot) \in \mathcal{V}(e)$ with

$\mathcal{V}(e):=\operatorname{conv}\left\{\left(\begin{array}{c}b_{1} \bar{k}_{1} \\ \vdots \\ b_{n_{u}} \bar{k}_{n_{u}}\end{array}\right)-\bar{\Delta} K e, b_{1}, \ldots, b_{n_{u}} \in\{ \pm 1\}\right\}$

where $\bar{k}_{i}:=\beta\left\|[\bar{\Delta}]_{i}\right\|, n_{u}$ denotes the dimension of the input space and, for a given set $\mathcal{A}$ and matrix $A, \operatorname{conv} \mathcal{A}$ and $[A]_{i}$ denote the convex hull of $\mathcal{A}$ and the ith row of $A$, respectively. 
Assumption 8 (Feasibility of the Auxiliary Law): The control law (11) is feasible in a neighborhood of $e=0$, i.e., $k\left(R(t), 0, v_{d}(t)\right) \in \operatorname{int}(\mathcal{U}), \forall t \in[0, \infty)$.

Assumption 8 guarantees that once $e=0$, i.e., the vehicle entered the $\epsilon$-tube around the path, a feasible input that keeps the error equal zero always exists. Since, due to the timevarying nature of $k\left(R(t), 0, v_{d}(t)\right)$, this assumption can be difficult to be a priori verified, later in this Section IV-D an alternative version is presented.

\section{B. MPC for Trajectory-tracking}

In this section Problem 1 is solved using Theorem 1 together with the control law from Proposition 1.

Corollary 1 (MPC for Trajectory-tracking): Consider the constrained system (1) and the control problem Problem 1. Let $\bar{u}_{t r}^{*}\left([0, T] ; z, t_{i}\right)$ be the solution to the optimization problem $P_{t r}\left(z, t_{i}\right)$ described by

$$
\begin{aligned}
& J_{t r}^{*}\left(z, t_{i}\right)=\min _{\bar{u}_{t r}([0, T])} J_{t r}\left(z, t_{i}, \bar{u}_{t r}([0, T])\right) \\
& \text { s.t. } \\
& \dot{\bar{p}}(\tau)=\bar{R}(\tau) \bar{v}(\tau) \\
& \dot{\bar{R}}(\tau)=\bar{R}(\tau) \Omega(\bar{\omega}) \\
& \bar{e}(T) \in \mathcal{E}_{f},(\bar{p}(0) \quad \bar{R}(0))=z \\
& \bar{e}(\tau)=\bar{R}(\tau)^{\prime}\left(\bar{p}(\tau)-p_{d}\left(t_{i}+\tau\right)\right)-\epsilon \quad \forall \tau \in[0, T] \\
& \bar{u}_{t r}(\tau)=\left(\bar{v}_{1}(t) \quad \bar{\omega}(\tau)^{\prime}\right)^{\prime} \in \mathcal{U} \\
& \forall \tau \in[0, T] \\
& \forall \tau \in[0, T] \\
& \forall \tau \in[0, T]
\end{aligned}
$$

with

$$
\begin{aligned}
& J_{t r}\left(z, t_{i}, \bar{u}([0, T])\right):=\int_{0}^{T}\|\bar{e}\|_{Q}^{2} \\
& \quad+\|\left(\Delta \bar{u}_{t r}-\bar{R}^{\prime} \dot{p}_{d}\left(t_{i}+\tau\right)\left\|_{O}^{2} d \tau+a_{2}\right\| \bar{e}(T) \|^{2}\right.
\end{aligned}
$$

where $a_{2}=\frac{\lambda_{\max }\left(Q+K^{\prime} O K\right)}{2 \lambda_{\min }(K)}, Q$ and $O$ are a positive-definite matrix and a positive-semidefinite matrix, respectively, with suitable dimensions, and $\mathcal{E}_{f}(\alpha)=\left\{e: \frac{1}{2} e^{\prime} e \leq \alpha\right\}$, for any positive constant $\alpha$ and positive-definite matrix $K$ such that $k(R(t), e, v(t)) \in \mathcal{U}, \quad \forall e \in \mathcal{E}_{f}(\alpha), \quad \forall t \in[0, \infty)$. If Assumptions 7-8 hold and the optimization problem $P_{t r}((p(t), R(t)), t)$ is feasible at initial time $t=0$, then the sampled-data MPC feedback law defined by

$u_{M P C, t r}(t)=\bar{u}_{t r}^{*}\left(t-t_{i} ;\left(p\left(t_{i}\right), R\left(t_{i}\right)\right), t_{i}\right), \forall t \in\left[t_{i}, t_{i+1}\right)$

solves Problem 1 and the region of attraction coincides with the set of initial positions and headings of the vehicle for which $P_{t r}((p(0), R(0)), 0)$ admits a feasible solution.

Due to space constraints the full proof is omitted, although note that the trajectory-tracking problem can be seen as a specialization of the path-following problem where $\dot{p}_{d}=$ $\frac{\partial p_{d}(\gamma(t))}{\partial \gamma} \dot{\gamma}_{d}$ and the proof of Corollary 1 follows similarly to the proof of Corollary 2 .

\section{MPC for Path-Following}

Corollary 2 (MPC for path-following): Consider the constrained system (1) and the control problem Problem 2. Let $\bar{u}_{p f}^{*}([0, T] ; z)$ be the solution to the optimization problem $P_{p f}(z)$ described by

$$
\begin{aligned}
& J_{p f}^{*}(z)=\min _{\bar{u}_{p f}([0, T])} J_{p f}\left(z, \bar{u}_{p f}([0, T])\right) \\
& \text { s.t. } \\
& \dot{\bar{p}}(\tau)=\bar{R}(\tau) \bar{v}(\tau) \\
& \forall \tau \in[0, T] \\
& \dot{\bar{R}}(\tau)=\bar{R}(\tau) \Omega(\bar{\omega}) \\
& \forall \tau \in[0, T] \\
& \bar{e}(T) \in \mathcal{E}_{f}, \quad(\bar{p}(0) \quad \bar{R}(0) \quad \bar{\gamma}(0))=z \\
& \bar{e}(\tau)=\bar{R}(\tau)^{\prime}\left(\bar{p}(\tau)-p_{d}(\gamma(\tau))\right)-\epsilon \\
& \forall \tau \in[0, T] \\
& \bar{u}_{p f}(\tau)=\left(\begin{array}{lll}
\bar{v}_{1}(t) & \left.\bar{\omega}(t)^{\prime} \quad \dot{\bar{\gamma}}(\tau)\right)^{\prime}
\end{array}\right. \\
& \dot{\bar{\gamma}}(\tau) \in \mathcal{G}, \quad\left(\bar{v}_{1}(t) \quad \bar{\omega}(t)^{\prime}\right)^{\prime} \in \mathcal{U} \\
& \forall \tau \in[0, T] \\
& \forall \tau \in[0, T] \\
& \forall \tau \in[0, T]
\end{aligned}
$$

with

$$
\begin{aligned}
& J_{p f}\left(z, \bar{u}_{p f}([0, T])\right):=\int_{0}^{T}\|\bar{e}\|_{Q}^{2}+\left\|\Delta \bar{u}_{p f}-\bar{R}^{\prime} \frac{\partial p_{d}}{\partial \gamma} \dot{\bar{\gamma}}\right\|_{O}^{2} \\
& \quad+\left\|\dot{\bar{\gamma}}-\dot{\gamma}_{d}\right\|_{o}^{2} d \tau+a_{2}\|\bar{e}(T)\|^{2}
\end{aligned}
$$

where $a_{2}=\frac{\lambda_{\max }\left(Q+K^{\prime} O K\right)}{2 \lambda_{\min }(K)}, Q$ and $O$ are a positivedefinite matrix and a positive-semidefinite matrix, respectively, with suitable dimensions, $o \in \mathbb{R}_{>0}$, and $\mathcal{E}_{f}(\alpha)=\left\{e:(1 / 2) e^{\prime} e \leq \alpha\right\}$, for any positive constant $\alpha \in \mathbb{R}_{\geq 0}$ and positive-definite matrix $K$ such that $k(R(t), e, v(t)) \in \mathcal{U}, \forall e \in \mathcal{E}_{f}(\alpha), \forall t \in[0, \infty)$. If Assumptions 7-8 hold and the optimization problem $P_{p f}(z(t))$, with $z(t)=\left(\begin{array}{lll}p(t) & R(t) & \gamma(t)\end{array}\right)$, is feasible at initial time $t=0$, then sampled-data MPC feedback law defined by

$$
u_{M P C, p f}(t)=\bar{u}_{p f}^{*}\left(t-t_{i} ; z\left(t_{i}\right)\right), \forall t \in\left[t_{i}, t_{i+1}\right),
$$

solves Problem 1 and the region of attraction coincides with the set of $z$ for which $P_{t r}(z)$ admits a feasible solution.

Proof: In this proof we first rewrite the error system (10), and the associated input constraint set, in a new input coordinates system, then Theorem 1 with the auxiliary control law obtained from $k(\cdot)$, is applied to stabilize the redefined error system. Performing the input coordinates transformation $v=\phi(u):=\Delta u-R^{\prime} \frac{\partial p_{d}}{\partial \gamma}(t) \dot{\gamma}$ the error system (10) becomes $\dot{e}=-\Omega(t) e+v, t \in[0, \infty)$, which satisfies Assumption 1, and the input constraint set $\mathcal{U}$ and the control law $k(\cdot)$ became $\mathcal{U}_{e}:=\{v: u \in \operatorname{int}(\mathcal{U}), \dot{\gamma} \in \mathcal{G}\}=$ $\left\{v: \bar{\Delta}\left(v+R^{\prime} \frac{\partial p_{d}}{\partial \gamma} \dot{\gamma}\right) \in \operatorname{int}(\mathcal{U}), \quad \dot{\gamma} \in \mathcal{G}\right\}$ and $v=k_{e}(e):=-K e$, respectively. Defining the auxiliary control law as $v=k_{e}(e)$ and $\dot{\gamma}=\dot{\gamma}_{d}$, which is feasible in a neighborhood of $e=0$ by Assumption 8, we have that $0 \in \operatorname{int}\left(\mathcal{U}_{e}\right)$ and, thus, Assumption 2 is satisfied. Moreover, the stage cost $l(e, v, \dot{\gamma}):=\|e\|_{Q}^{2}+\|v\|_{R}^{2}+\left\|\dot{\gamma}-\dot{\gamma}_{d}\right\|_{o}^{2}$ satisfies Assumption 3 and the auxiliary control law with the Lyapunov function $V(\cdot)$ satisfies Assumption 4 with $k_{1}=k_{2}=0.5, a=2, k_{3}=\lambda_{\min }(K)$, and $\mathcal{X}_{D}=\mathbb{R}^{2}$ or, for the 3-D case, $\mathcal{X}_{D}=\mathbb{R}^{3}$. Note that, although the auxiliary control law identically sets $\dot{\gamma}=\dot{\gamma}_{d}$, this does not apply to the resulting MPC controller, which will optimally choose the input $\dot{\gamma}$. Combining stage cost with the auxiliary control law, Assumption 5 is satisfied with $a_{2}=\lambda_{\max }\left(Q+K^{\prime} O K\right)$, $v=2$, and $\mathcal{X}_{l}=\mathbb{R}^{2}$ or, for the 3-D case, $\mathcal{X}_{l}=\mathbb{R}^{3}$. 
Applying Theorem 1 we obtain Corollary 2, where we made explicit the dependence on the vehicle model.

Remark 2: Corollary 1 and Corollary 2 still hold if we enforce the error vector $e$ to lie inside a connected set containing the origin in its interior. This can be of interest for some applications where we would like to enforce the closed-loop trajectory of the position of the vehicle to be bounded inside an outer tube around the desired path.

\section{Computation of the Terminal Set}

In order to verify the feasibility of the auxiliary control law in Assumption 8 along all the time $t$, we use the bound from Assumption 7, and we introduce the following assumption:

Assumption 9: The input constraint set $\mathcal{U}$ is a closed basic semi-algebraic set that contains $\mathcal{V}(0)$ in its interior.

Note that Assumption 9 implies Assumption 8 and can be considered its robustified version, thus more conservative.

Moreover, note that $\left\{e: \mathcal{V}_{\mathcal{B}}(e) \subseteq \mathcal{U}\right\}$ is a basic semi-algebraic set that inner approximates $\left\{e: k_{t r}(e)=-K e \in \mathcal{U}_{e}\right\}=\left\{e: k\left(R, e, \dot{p}_{d}\right) \in \mathcal{U}\right\}$, which from Assumption 9, is never empty and, following Remark 1, it can be used to efficiently solve the optimization problem (9) and to compute the desired terminal set.

Remark 3: It is worth noting that, for the case of vehicles with unconstrained inputs, i.e. $\mathcal{U}=\mathbb{R}^{n}$, any choice of $\alpha>0$ is a feasible. Thus, the terminal set can be neglected, resulting in MPC controllers that globally solve the addressed motion control problems.

\section{Simulation Results}

This section illustrates the previous results with a models of a wheeled robot (2-D) and of an aero vehicle (3-D). For each vehicle and from different initial configurations, we simulate and compare the closed-loop trajectories of the system with the three proposed controllers, i.e., the auxiliary law from Proposition 1, the MPC trajectory-tracking controller from Corollary 1, and the MPC path-following controller from Corollary 2.

\section{A. 2-D case : Wheeled Robot}

Consider the constrained unicycle model (1) with $\mathcal{U}=\left\{\left(\begin{array}{ll}u & \omega\end{array}\right)^{\prime}: u \in[-3,3], \omega \in[-10,10]\right\}$. The desired trajectory is defined as $p_{d}(t)=\left(\begin{array}{ll}0.4 t & \sin 0.4 t\end{array}\right)^{\prime}$ and the auxiliary control law from Proposition 1 is chosen with $\epsilon=\left(\begin{array}{ll}0.2 & 0\end{array}\right)^{\prime}$ and $K=0.8 I_{2 \times 2}$, where for a given $n \in \mathbb{N}_{>0}$, we denote by $I_{n \times n} \in \mathbb{R}^{n \times n}$ the identity matrix with given dimensions. The trajectory satisfies Assumption 7 with $\begin{aligned} & \beta=\left\|\left(\begin{array}{cc}0.4 & 0.4\end{array}\right)^{\prime}\right\| \geq \| \\ & \text { Using }\end{aligned} \| \begin{array}{cc}\left(\begin{array}{cc}0.4 & 0.4 \cos 0.4 t\end{array}\right)^{\prime} \| & =\|\end{array}=\begin{aligned} & \left\|\dot{p}_{d}\right\| . \\ & \text { with }\end{aligned}$ $\mathcal{V}(e)=\operatorname{conv}\left\{\left(\begin{array}{l}b_{1} 0.5657 \\ b_{2} 2.8284\end{array}\right)-\bar{\Delta} 0.8 e, b_{1}, b_{2} \in\{-1,+1\}\right\}$ which satisfies Assumptions 8-9. The stage cost function is chosen with $Q=10 I_{2 \times 2}$ and $O=0.1 I_{2 \times 2}$, and the terminal set $\mathcal{E}_{f}=\left\{e: e^{\prime} e \leq 3.18\right\}$ is computed following the procedure proposed in Section IV-D.
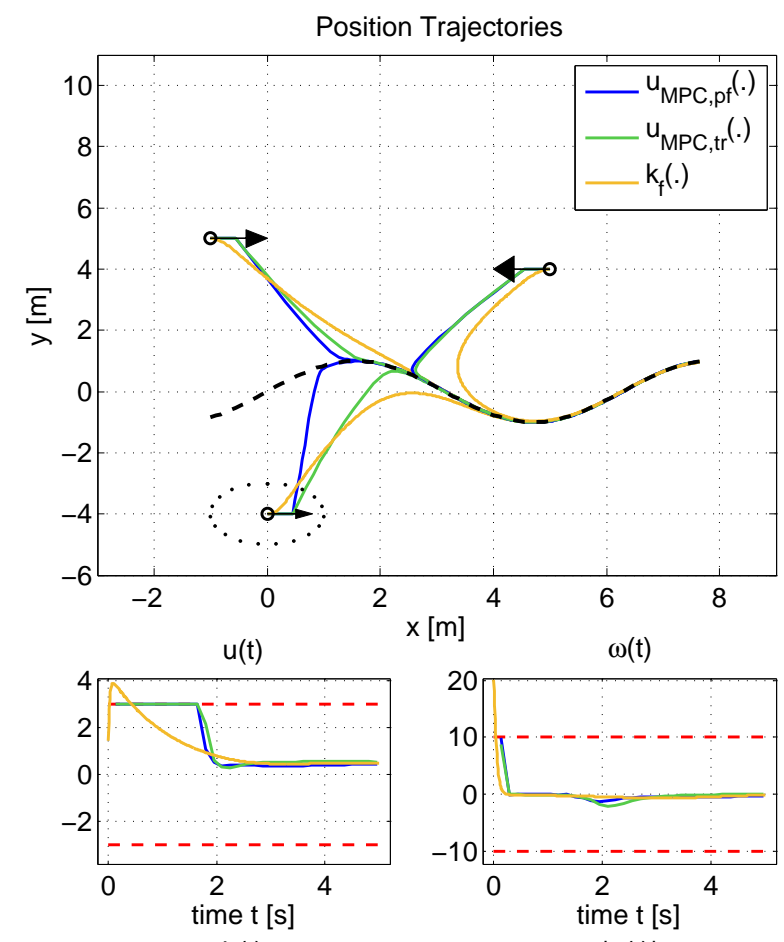

$b(t)$

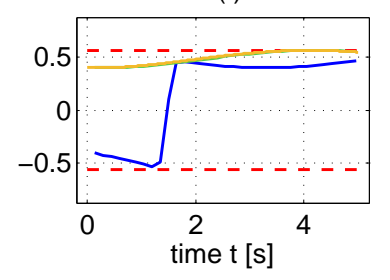

$|e(t)|$

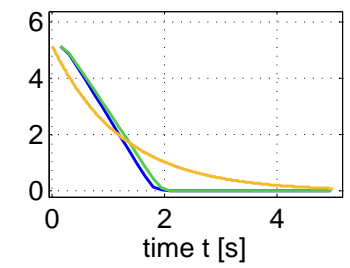

Fig. 1. Trajectories of the position of the wheeled robot in closed-loop with the proposed control laws for different initial positions and headings, which are by the arrows. The black dotted line represents the desired path. One initial configuration, identified with the dotted circle, is chosen and the details on control inputs, tracking error, and velocity of the desired point are displayed. In order to emphasize the direction of the desired position we define $b(t):=\operatorname{sign}\left(\dot{p}_{d}^{\prime}(t)[1,0]^{\prime}\right)\left\|\dot{p}_{d}(t)\right\|$.

For the path-following controller, the trajectory is parametrized with the parameter $\gamma$ as $p_{d}(\gamma)=\left(\begin{array}{ll}\gamma & \sin \gamma\end{array}\right)^{\prime}$, and we chose the desired parameter speed $\dot{\gamma}_{d}=0.4 \in \mathcal{G}$, with $\mathcal{G}=[-1,1]$. Similarly to the trajectory-tracking case, Assumptions 7-9 hold and the MPC path-following and the stage cost is choose with $Q=10 I, O=0.1 I_{2 \times 2}$, $o=2$. Numerical results are presented in Fig. 1 where, for simulation purposes, the system is discretized with a discretization step of 0.15 seconds. The horizon length is chosen as $T=1.5$ seconds. Note that, contrarily to the auxiliary control law and to the MPC trajectory-tracking controller $u_{t r}(\cdot)$ where $\dot{p}_{d}(t)$ is a priori fixed, in the MPC path-following law we have $\dot{p}_{d}(t)=\frac{\partial \dot{p}_{d}}{\gamma} \dot{\gamma}$ where $\dot{\gamma}$ is an input that can be controlled. As effect, during the initial phase, the desired position goes in the direction of the 


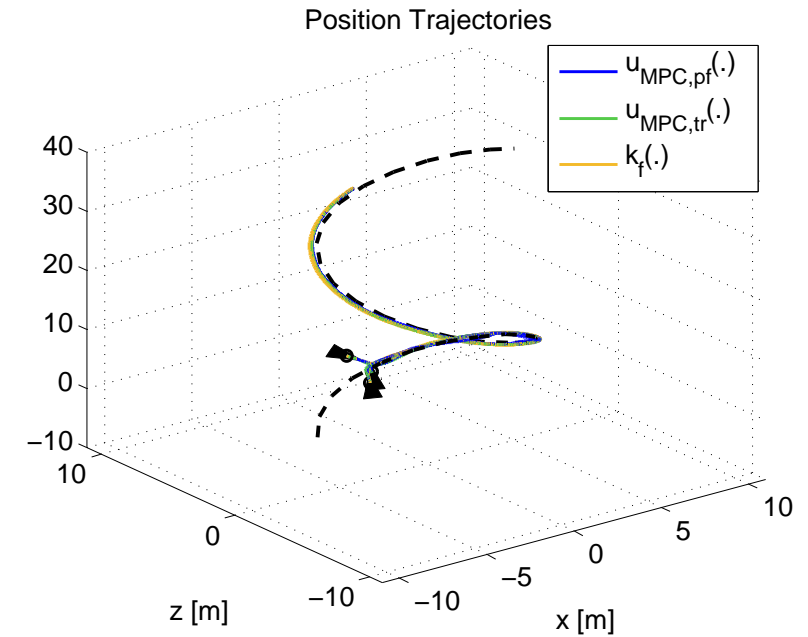

Fig. 2. Using different initial positions and headings of the vehicle, represented by the arrows, the trajectories of the position of the vehicle in closed-loop with the proposed control laws are displayed. With the black dotted line we identify the desired path.

negative $x$ in order to decrease the error $e$ "waiting" for the vehicle to approach the desired path.

\section{B. 3-D case : Aero Vehicle}

Consider the constrained Aero Vehicle model (1) with $\mathcal{U}=\left\{\left(\begin{array}{ll}u & \omega^{\prime}\end{array}\right)^{\prime}: u \in[-3,3],-\bar{\omega} \leq \omega \leq \bar{\omega}\right\}$, $\bar{\omega}=\left(\begin{array}{lll}10 & 10 & 0\end{array}\right)^{\prime}$, where we are able to only control forward velocity, roll and pitch (i.e. the yaw is identically set to zero). The desired trajectory is defined as $p_{d}(t)=5(\sin (0.08 t) \quad \cos (0.08 t) \quad 0.08 t)^{\prime}$ and the auxiliary law from Proposition 1 is chosen with $\epsilon=\left(\begin{array}{ccc}-0.2 & 0 & -0.2\end{array}\right)^{\prime}$ and $K=I_{3 \times 3}$. The trajectory satisfies Assumption 7 with $\beta=\left\|\left(\begin{array}{lll}0.4 & 0.4 & 0.4\end{array}\right)\right\|$. Using $\beta$ and the chosen $\epsilon$ we obtain $\mathcal{V}(e)=\operatorname{conv}\left\{\left(\begin{array}{lll}b_{1} 1.4142 & b_{2} 2.5 & b_{3} 5\end{array}\right)^{\prime}-\bar{\Delta} e\right.$, $\left.b_{1}, b_{2}, b_{3} \in\{-1,+1\}\right\}$ which satisfies Assumptions 8-9. The stage cost function is chosen with $Q=10 I_{3 \times 3}$ and $O=I_{4 \times 4}$, and the terminal set $\mathcal{E}_{f}=\left\{e: e^{\prime} e \leq 1.7\right\}$ is computed following the procedure proposed in Section IV-D.

For the path-following controller the trajectory is parametrized with the parameter $\gamma$ as $p_{d}(\gamma)=(\cos \gamma,-\sin \gamma, \gamma)^{\prime}$, and we chose the desired speed $\dot{\gamma}_{d}=0.08 \in \mathcal{G}$, with $\mathcal{G}=[-1,1]$.

Similarly to the trajectory-tracking case, Assumptions 79. The stage cost function is chosen with $Q=10 I_{3 \times 3}$, $O=I_{4 \times 4}, o=1$. Numerical results are presented in Fig. 2 where, for simulation purposes, the system is discretized with a discretization step of 0.1 seconds. The horizon length is chosen as $T=1$ seconds.

\section{CONCLUSION}

The motion control problem of underactuated vehicles is addressed and two MPC controllers, one for trajectorytracking and one for path-following, that guarantee asymptotic convergence of the position of the vehicle to a tube centered around the desired path are presented. Allowing an asymptotic tracking error, we are able to compute the terminal set and the terminal law avoiding linearization procedures. This results in MPC strategies where, for a given horizon length, the size of the region of attraction is only limited by the size of the constraints leading to global solutions for the case of unconstrained systems.

\section{REFERENCES}

[1] A. Aguiar and J. Hespanha, "Position tracking of underactuated vehicles," in Proc. of the American Control Conf., vol. 3, pp. 19881993, IEEE, 2003.

[2] R. Fierro and F. Lewis, "Control of a nonholonomic mobile robot: backstepping kinematics into dynamics," in Proc. of the 34th IEEE Conf. on Decision and Control, vol. 4, pp. 3805-3810, IEEE, 1995.

[3] B. D'Andrea-Novel, G. Bastin, and G. Campion, "Dynamic feedback linearization of nonholonomic wheeled mobile robots," in Proc. IEEE International Conf. on Robotics and Automation, pp. 2527-2532, IEEE Comput. Soc. Press, 1992.

[4] G. Oriolo, A. De Luca, and M. Vendittelli, "WMR control via dynamic feedback linearization: design, implementation, and experimental validation," IEEE Transactions on Control Systems Technology, vol. 10, pp. 835-852, Nov. 2002.

[5] C. Samson and K. Ait-Abderrahim, "Feedback control of a nonholonomic wheeled cart in Cartesian space," in Proc. IEEE International Conference on Robotics and Automation, pp. 1136-1141, IEEE Comput. Soc. Press, 1991.

[6] H. Chen and F. Allgöwer, "A Quasi-Infinite Horizon Nonlinear Model Predictive Control Scheme with Guaranteed Stability," Automatica, vol. 34, pp. 1205-1217, Oct. 1998.

[7] D. Mayne, J. Rawlings, C. Rao, and P. Scokaert, "Constrained model predictive control: Stability and optimality," Automatica, vol. 36, pp. 789-814, June 2000.

[8] F. A. Fontes, "A general framework to design stabilizing nonlinear model predictive controllers," Systems \& Control Letters, vol. 42, pp. 127-143, Feb. 2001.

[9] R. Findeisen and F. Allgöwer, "The quasi-infinite horizon approach to nonlinear model predictive control.," Nonlinear and Adaptive Control, 2003.

[10] R. Findeisen, L. Imsland, F. Allgower, and B. A. Foss, "State and output feedback nonlinear model predictive control: An overview," European journal of control, vol. 9, no. 2-3, pp. 190-206, 2003.

[11] T. Faulwasser, Optimization-based Solutions to Constrained Trajectory-tracking and Path-following Problems. $\mathrm{PhD}$ thesis, Otto-von-Guericke-Universität Magdeburg, 2012.

[12] T. Faulwasser and R. Findeisen, Nonlinear Model Predictive PathFollowing Control, vol. 384 of Lecture Notes in Control and Information Sciences. Springer Berlin / Heidelberg, 2009.

[13] L. Magni and R. Scattolini, "Tracking of non-square nonlinear continuous time systems with piecewise constant model predictive control," Journal of Process Control, vol. 17, pp. 631-640, Sept. 2007.

[14] L. Magni, G. De Nicolao, and R. Scattolini, "Output feedback and tracking of nonlinear systems with model predictive control," Automatica, vol. 37, pp. 1601-1607, Oct. 2001.

[15] S. Yu, X. Li, H. Chen, and F. Allgöwer, "Nonlinear Model Predictive Control for Path Following Problems," in Proc. of the Nonlinear Model Predictive Control Conference, 2012.

[16] D. Gu and H. Hu, "Receding horizon tracking control of wheeled mobile robots," IEEE Transactions on Control Systems Technology, vol. 14, pp. 743-749, July 2006.

[17] A. Alessandretti, M. N. Zeilinger, A. P. Aguiar, and C. N. Jones, "A Nonlinear Model Predictive Control Scheme with Guaranteed Stability using a Given Exponentially Stabilizing Law," tech. rep., url: web.ist.utl.pt/andrea.alessandretti/techmpces13.pdf, 1012.

[18] J. Sturm, "Using SeDuMi 1.02, a Matlab Toolbox for Optimization over symmetric cones," Optimization methods and software, 1999.

[19] R. Tütüncü, K. Toh, and M. Todd, "Solving semidefinite-quadraticlinear programs using SDPT3," Mathematical programming, pp. 1-31, 2003.

[20] J. Lofberg, "YALMIP : a toolbox for modeling and optimization in MATLAB," IEEE International Conf. on Robotics and Automation, pp. 284-289, 2004. 